\title{
THE MONASH UNIVERSITY OBSERVATORY: EQUIPMENT AND RESEARCH
}

\author{
J.L. Innis, K. Thompson and D.W. Coates \\ Department of Physics, Monash University, Wellington Road, \\ Clayton, Australia
}

\section{INTRODUCTION}

The Monash Observatory, located at Mount Burnett, Victoria, seventy kilometres east of the city of Melbourne, at a latitude of $38^{\circ} \mathrm{S}$ has a $0.45-\mathrm{m}$ Cassegrainian and a $0.25-\mathrm{m}$ Newtonian telescope. Both are equipped for photoelectric photometry, with microcomputer-based data logging systems. The $0.45-\mathrm{m}$ has recently replaced the observatory's original $0.4-\mathrm{m}$ Newtonian. Our site is near the coast, and has less than $20 \%$ photometric weather. Access to the telescopes and facilities of Mount Stromlo and Siding Spring Observatories (MSSSO) increases our observing time and the scope of our research. For a description of the Monash Observatory at mid-1984 see Coates et a1, (1984).

\section{RESEARCH}

While our original work was on short period pulsating stars, our current research interest is the study of chromospherically active stars. We commenced work on these stars in 1979, initially concentrating on southern RS CVn candidates, searching for broadband variations due to starspots, determining rotation rates, and searching for light curve variability due to changes in spot sizes and/or locations. In 1983 we began spectroscopic observations with the telescopes of MSSSO to study the binary nature of these stars and the changes in the $\mathrm{Ca}$ II and $\mathrm{H} \alpha$ emission lines. In 1984 we began collaborative work with radioastronomers from the Australian Commonwealth Scientific and Industrial Research Organisation (CSIRO), Division of Radiophysics (Drs. O.B. Slee, G.J. Nelson and

A.E. Wright).

Light curves and other data which we have obtained illustrate the rapidity of the changes in the properties of spots on these stars (eg: Innis et al, 1984, and work to appear in Proc. Astron. Soc. Aust.). For example in figure 1 we show $V$ light curves for the rapidly rotating single star AB Dor, for 1984 February-March, 1984

J. B. Hearnshaw and P. L. Cottrell (eds.), Instrumentation and Research Programmes for Small Telescopes, 293-294.

(C) 1986 by the IAU. 
November, and 1985 February. The first two curves were obtained with the $0.25-\mathrm{m}$ telescope, while the third, which shows a small flare near phase 0.8 , was obtained at Siding Spring Observatory. The changes in the light curves are quite marked. Rucinski (1983) reported even faster changes for this star, on the order of days.

Our recent work on $A B$ Dor suggests that it is a young star, just arriving on the main sequence. It appears to be very similar to the rapidly rotating Pleiades $\mathrm{K}$ dwarfs (Van Leeuwen and Alphenaar, 1983). We are continuing the analysis.

\section{ACKNOWLEDGEMENTS}

We acknowledge the contributions of former graduate students Len Halprin and Terry Moon to the development of the Monash Observatory. We thank the staff of the Department of Physics Electronic and Mechanical Workshops for excellent technical support. We also thank MSSSO for access to their telescopes and facilities. The Monash Observatory is supported by the Australian Research Grants Scheme, a Monash University Special Research Grant, the Monash/CSIRO Collaborative fund, and the Department of Physics. John Innis is supported by an Australian Commonwealth Postgraduate Research Award.

\section{REFERENCES}

Coates, D.W., Thompson, K., Innis, J.L. and Moon, T.T., 1984. In Advances in Photoelectric Photometry Vol. 2, p. 74, eds. Wolpert, R.C., and Genet, R.M., Fairborn, Ohio.

Innis, J.L., Coates, D.W. and Thompson, K., 1984. Proc. Astron. Soc. Aust. 5, 540 .

Rucinski, S.M., 1983. Astron. Astrophys. Supp1. Ser., 52, 281. Van Leeuwen, F. and Alphenaar, P., 1983. IAU Col1. No. 71, Activity in Red Dwarf Stars, p. 189, eds. Byrne, P.B. and Rodono, M., Dordrect, Reide1.
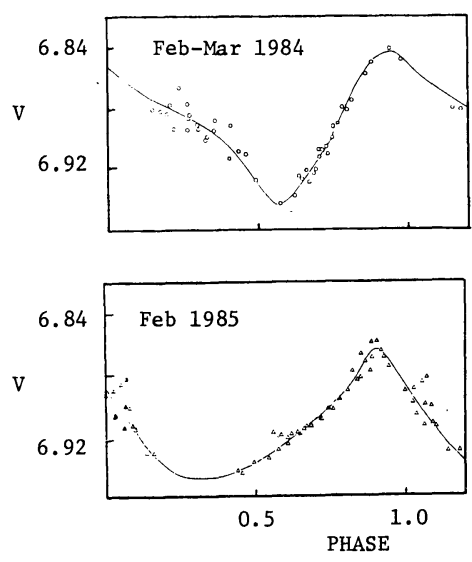

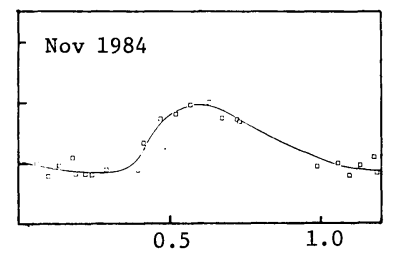

Figure 1. V 1ight curves for $A B$ Dor (HD 36705) for the dates shown. 